\title{
Ellipse-based DCO-OFDM for visible light communications
}

\author{
Tianqi Mao*, Zhaocheng Wang, Qi Wang, Linglong Dai \\ Tsinghua National Laboratory for Information Science and Technology (TNList), Department of Electronic Engineering, Tsinghua University, Beijing 100084, \\ China
}

\section{A R T I C L E I N F O}

\section{Article history:}

Received 26 August 2015

Received in revised form

24 September 2015

Accepted 5 October 2015

\section{Keywords:}

Visible light communication

OFDM

LED nonlinearity

PAPR

Performance optimization

\begin{abstract}
A B S T R A C T
Ellipse-based DC-biased optical orthogonal frequency division multiplexing (E-DCO-OFDM) is proposed for visible light communications (VLC), which achieves a significant peak-to-average power ratio (PAPR) reduction, thus enhancing the overall performance when light-emitting diode (LED) nonlinearity is considered. In E-DCO-OFDM, the real-valued output of OFDM is modulated onto an ellipse, whereby only the imaginary part of the complex point on the ellipse is transmitted. Although the PAPR of E-DCO-OFDM decreases as the ratio of major radius to minor radius becomes larger, it may be more vulnerable to the effect of noise, leading to the performance loss. Therefore, the relationship between the system performance and the critical parameters in E-DCO-OFDM, such as the ratio between the major and minor radius of the ellipse, is investigated. Meanwhile, simulations demonstrate that E-DCO-OFDM adopting the optimal parameters achieves a considerable signal-to-noise ratio (SNR) gain over the conventional DCO-OFDM.
\end{abstract}

(c) 2015 Elsevier B.V. All rights reserved.

\section{Introduction}

Visible light communication (VLC) is emerging as a solution to deal with the scarcity of radio spectrum for wireless communication systems, which draws great interests due to its security, safety for human health, and complementarity to radio frequency (RF) systems [1]. In VLC, white light-emitting diodes (LEDs) are employed to perform both data transmission and illumination at the same time [2]. In order to avoid inter-symbol interference (ISI) as well as to increase the data rate, orthogonal frequency division multiplexing (OFDM) is introduced [3-5]. Since intensity modulation with direct detection (IM/DD) is often applied in VLC systems, only unipolar real-valued signals can be transmitted by LEDs. There exist several approaches to deal with this issue, including DC-biased optical OFDM (DCO-OFDM) [6], asymmetric clipped optical OFDM (ACO-OFDM) [7], and pulse-amplitude-modulated discrete multitone (PAM-DMT) [8], where Hermitian symmetry is imposed on the subcarriers in frequency domain in order to generate real-valued signals after inverse fast Fourier transform (IFFT) [9]. In DCO-OFDM, a moderate DC-bias is added to make the signal non-negative [10]. For ACO-OFDM and PAM-DMT, no DC-bias is needed, since only half of the subcarriers are used in ACO-OFDM, and PAM-DMT merely modulates the imaginary part of each subcarrier, both generating unipolar outputs after clipping [9].

\footnotetext{
* Corresponding author.

E-mail addresses: mtq15@mails.tsinghua.edu.cn (T. Mao), zcwang@tsinghua.edu.cn (Z. Wang), qiwang11@mails.tsinghua.edu.cn (Q. Wang), daill@tsinghua.edu.cn (L. Dai).
}

DCO-OFDM suffers from high peak-to-average power ratio (PAPR) problem, causing severe distortion due to the nonlinear transfer characteristics of LEDs. To reduce the PAPR, various schemes have been proposed. In [11], tone injection (TI) is performed using semidefinite relaxation techniques, achieving a significant PAPR reduction with high complexity. While in [12], exponential nonlinear companding is proposed to reduce the PAPR considerably with the sacrifice of the performance. Besides that, a pilot-assisted method is employed in [13]. It outperforms the conventional selective mapping (SLM) scheme, but suffers from performance loss and low data rate due to the requirement of side information. Active constellation extension (ACE) is also applied to DCO-OFDM in [14], which is an efficient technique with high computational complexity.

Constant-envelop OFDM (CE-OFDM) [15] can achieve $0 \mathrm{~dB}$ PAPR of OFDM with relatively satisfactory bit error rate (BER) performance, whereby the outputs of IFFT are modulated onto the phase to generate complex signals with constant envelop, and the complex signals are transmitted. Since only unipolar real-valued signals can be transmitted by LEDs, CE-OFDM cannot be directly applied to VLC systems. To adopt the concept of constant envelop in DCO-OFDM, an ellipse-based DCO-OFDM (E-DCO-OFDM) is proposed in this paper, whereby real-valued signals, multiplied by a scaling factor, are modulated onto an ellipse, and only the imaginary value from the corresponding point on the ellipse is transmitted by LED emitters. In E-DCO-OFDM, the lengths of major and minor radius are critical parameters which influence the overall system performance. Therefore, the optimal ratio between the major and minor radius of the ellipse are obtained by 
theoretical analysis, and simulations are performed to validate the optimization results. When considering the nonlinear transfer characteristics of LEDs, E-DCO-OFDM could improve the overall performance significantly compared with DCO-OFDM.

The rest of this paper is organized as follows. Section 2 describes the system model of the proposed E-DCO-OFDM. Section 3 shows the theoretical analysis of the overall performance of E-DCO-OFDM. In Section 4, the performances of E-DCO-OFDM and DCO-OFDM are compared via simulations, which validates our analytical results. Finally, our conclusions are drawn in Section 5.

\section{System model of the proposed E-DCO-OFDM}

The system model of E-DCO-OFDM is presented in Fig. 1. Firstly, binary bits are modulated using quadrature amplitude modulation (QAM), and the frequency domain signals $\mathbf{X}=$ $\{X[0], X[1], X[2], \ldots, X[N-1]\}$ are constrained by Hermitian symmetry, which can be described as

$X[k]=X^{*}[N-k], \quad k=1,2, \ldots, N / 2-1$,

where $N$ is the number of subcarriers. $X[0]$ and $X[N / 2]$ are set to zero. After that, a $N$-point IFFT operation is performed on $\mathbf{X}$, generating realvalued signals $\quad \mathbf{x}=\left\{x_{D C O}[0], x_{D C O}[1], x_{D C O}[2], \ldots, x_{D C O}[N-1]\right\}$. The time domain sequence $\mathbf{x}$ is then modulated onto an ellipse, which is presented in Fig. 2. The phase information $\Theta=$ $\{\theta[0], \theta[1], \theta[2], \ldots, \theta[N-1]\}$ of the ellipse transform can be described as

$\theta[n]=\beta x_{D C O}[n]=\arctan \left(\frac{y[n]}{x[n]}\right), \quad n=0,1,2, \ldots, N-1$.

In (2), $\beta$ is a scaling factor defined as $\frac{\pi}{4 \sigma_{x} \times \gamma}$, constraining $\theta[n]$ for $n=0,1,2, \ldots, N-1$ mostly within $[-\pi / \gamma, \pi / \gamma]$, where $\sigma_{x}$ is the standard deviation of $x_{D C O}[n]$, and $\gamma$ is a real number larger than 2 so that the complex signals on the ellipse can be uniquely determined by their imaginary parts. $(x[n], y[n])$ is the coordinate of the corresponding point on an ellipse generated by $\theta[n]$, which follows:

$\frac{x[n]^{2}}{a^{2}}+\frac{y[n]^{2}}{b^{2}}=1$,

where $a$ and $b$ denote the major and minor radius if foci of the ellipse are on the real axis. Thus $(x[n], y[n])$ can be calculated as

$x[n]=\sqrt{\frac{1}{1 / a^{2}+\tan ^{2}(\theta[n]) / b^{2}}}$,

and

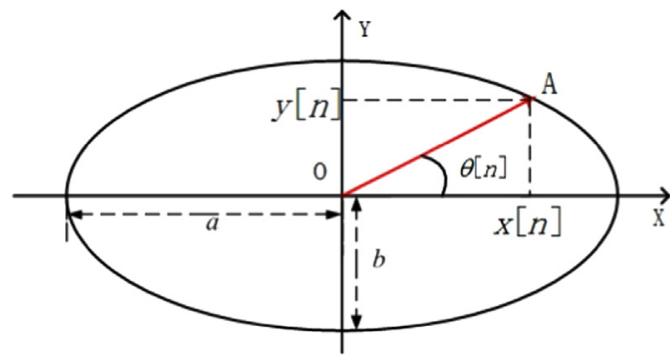

Fig. 2. Ellipse illustration of the proposed E-DCO-OFDM.

$y[n]=\tan (\theta[n]) \sqrt{\frac{1}{1 / a^{2}+\tan ^{2}(\theta[n]) / b^{2}}}$.

In E-DCO-OFDM, only the imaginary part $\{y[n]\}_{n=0}^{N-1}$ needs to be transmitted. After the ellipse transform, the cyclic prefix (CP) is inserted to eliminate the effect of ISI. The resulted signals are then fed into a digital-to-analog converter (D/A) to generate the analog signals. Finally, DC-bias is added using a Bias-T module to make the signal unipolar before sending it to the LED for transmission.

While at the receiver, the inverse operations are performed, including passing through an analog-to-digital converter (A/D), removing $\mathrm{DC}$-bias and $\mathrm{CP}$, the inverse ellipse transform, a $\mathrm{N}$-point FFT and QAM demapping. The inverse transform taken by the received signals $\left\{r_{y}[n]\right\}_{n=0}^{N-1}$ can be formulated as

$r_{x}[n]=a \sqrt{\left(1-\frac{r_{y}[n]^{2}}{b^{2}}\right)}, \quad n=0,1,2, \ldots, N-1$,

where $r_{y}[n]$ equals the signal of $y[n]$ plus the noise and distortions. After that, the estimation of $x_{D C O}[n]$, denoted as $\tilde{x}_{D C O}[n]$, is calculated by

$\tilde{x}_{D C O}[n]=\arctan \left(\frac{r_{y}[n]}{r_{x}[n]}\right) / \beta, \quad n=0,1,2, \ldots, N-1$.

\section{Performance optimization}

Assuming that $a / b$ is fixed, then $a$ is proportional to the $y[n]$ for $n=1,2, \ldots, N$. Since the transmitting energy is normalized, $a$ has no effect on the PAPR and BER of E-DCO-OFDM. Besides that, the value of $b$ is not relevant to the performance of E-DCO-OFDM. Therefore, it is concluded that the performance of E-DCO-OFDM is determined by the ratio of $a / b$ instead of the absolute values of $a$ and $b$ when the ellipse transform is considered. To optimize the

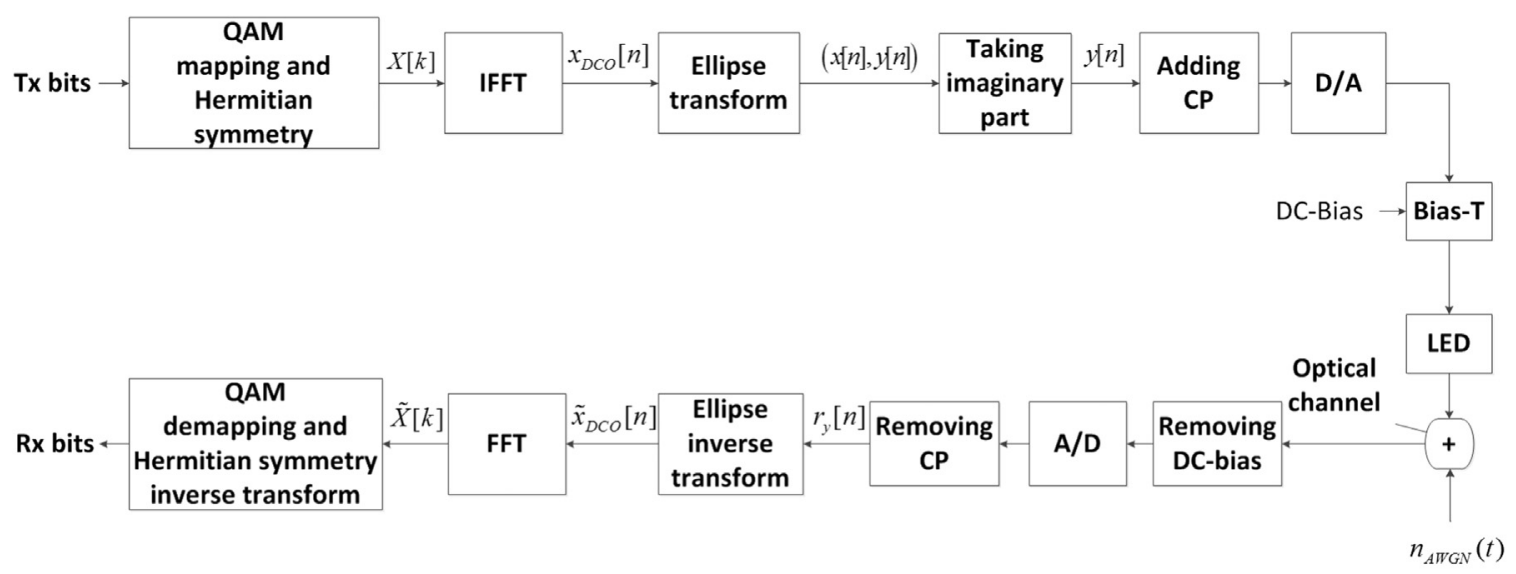

Fig. 1. E-DCO-OFDM system model. 
performance of E-DCO-OFDM, the PAPR as well as BER should be formulated as the function of $a / b$.

To demonstrate the relationship between the PAPR and $a / b$, the probability distribution function (PDF) of $\theta[n]$ for $n=$ $0,1,2, \ldots, N-1$ can be modeled as a zero-mean Gaussian distribution when $N$ is large enough [10]. Therefore, the PDF of $\theta[n]$ can be written as

$f_{\theta[n]}(v)=\frac{1}{\sigma \sqrt{2 \pi}} \exp \left(-\frac{v^{2}}{2 \sigma^{2}}\right)$,

where $\sigma^{2}$ is the variance of $\theta[n]$. Besides that, the PAPR of E-DCOOFDM can be formulated as

$P A P R=\frac{\max \left\{y^{2}[n]\right\}}{E\left\{y^{2}[n]\right\}}$,

where $E\{\cdot\}$ stands for the expectation operator. Since $\theta[n]$ is mostly constrained within $[-\pi / \gamma, \pi / \gamma]$, there exists an element of $\Theta$ which is close to $\pi / \gamma$. Thus the peak energy is approximately $b^{2} \tan ^{2}\left(\frac{\pi}{r}\right)$

$$
\begin{aligned}
& \frac{b^{2} \tan ^{2}\left(\frac{\pi}{\gamma}\right)}{b^{2} / a^{2}+\tan ^{2}\left(\frac{\pi}{\gamma}\right)} \text {, and we have } \\
& \text { PAPR } \approx \frac{\frac{b^{2} \tan ^{2}\left(\frac{\pi}{\gamma}\right)}{b^{2} / a^{2}+\tan ^{2}\left(\frac{\pi}{\gamma}\right)}}{\int_{-\pi / \gamma}^{\pi / \gamma} \frac{b^{2} \tan ^{2}(v)}{b^{2} / a^{2}+\tan ^{2}(v)} f_{\theta[n]}(v) d v} \\
& =\frac{\tan ^{2}\left(\frac{\pi}{\gamma}\right)}{\int_{-\pi / \gamma}^{\pi / \gamma} \frac{\tan ^{2}(v)\left(1+a^{2} \tan ^{2}\left(\frac{\pi}{\gamma}\right) / b^{2}\right)}{1+a^{2} \tan ^{2}(v) / b^{2}} f_{\theta[n]}(v) d v} .
\end{aligned}
$$

To figure out the monotonicity of PAPR which is a function of $k=a$ / $b$, we assume $S(k)=\frac{\tan ^{2}(v)\left(1+k^{2} \tan ^{2}\left(\frac{\pi}{\gamma}\right)\right)}{1+k^{2} \tan ^{2}(v)}$, the derivative of $S(k)$ can be formulated as

$S^{\prime}(k)=\frac{2 k \tan ^{2}(v)\left(\tan ^{2}\left(\frac{\pi}{\gamma}\right)-\tan ^{2}(v)\right)}{\left(k^{2} \tan ^{2}(v)+1\right)^{2}} \geq 0$.

Since $\tan ^{2}(v) \leq \tan ^{2}(\pi / \gamma)$ when $v \in[-\pi / \gamma, \pi / \gamma]$, it is obvious that $S^{\prime}(k)$ is non-negative, indicating that the denominator in (10) increases as the ratio of $a / b$ becomes larger, which could decrease the PAPR and make the system insensitive to the nonlinear distortions of LEDs. However, when the ratio of $a / b$ increases, E-DCOOFDM may be more vulnerable to the effect of noise. Thus, it is important to optimize the ratio of $a / b$ in order to improve the BER performance when the nonlinearity of LEDs is considered. Since $M$-QAM is employed in E-DCO-OFDM, we have [16]

$$
\begin{aligned}
B E R= & \frac{4(\sqrt{M}-1)}{\sqrt{M} \log _{2}(M)} Q\left(\sqrt{\frac{3 \log _{2} M}{M-1} S N R_{r}}\right) \\
& +\frac{4(\sqrt{M}-2)}{\sqrt{M} \log _{2}(M)} Q\left(3 \sqrt{\frac{3 \log _{2} M}{M-1} S N R_{r}}\right),
\end{aligned}
$$

where $S N R_{r}$ denotes the equivalent electronic signal-to-noise ratio (SNR) at the receiver, and $Q(\cdot)$ is defined as $Q(s)=\int_{s}^{\infty} \frac{1}{\sqrt{2 \pi}} \exp \left(-v^{2} / 2\right) d v$. In E-DCO-OFDM, there are totally four transforms, including the ellipse transform, the nonlinear transfer function of LEDs, the optical channel response, and the inverse ellipse transform. The ellipse transform can be formulated as
$\operatorname{Ell}(s)=\tan (\beta s) \sqrt{\frac{1}{1 / a^{2}+\tan ^{2}(\beta s) / b^{2}}}+D C_{t}$,

where $\operatorname{Ell}(\cdot)$ is the ellipse transform, and $D C_{t}$ is the DC bias. Since the nonlinear transfer characteristic of LEDs can be modeled as double-sided clipping by applying predistortion techniques [17], the simplified LED model in [11] is employed in our theoretical analysis, which can be presented as

$G(s)= \begin{cases}\lambda_{\max }, & s>\lambda_{\max } ; \\ s, & \lambda_{\min } \leq s \leq \lambda_{\max } \\ \lambda_{\min }, & s<\lambda_{\min },\end{cases}$

where $G(\cdot)$ denotes the simplified LED nonlinear model, and $\left[\lambda_{\min }, \lambda_{\max }\right]$ is the linear region of the LED. In (13), the value of $D C_{t}$ is set to $\left(\lambda_{\min }+\lambda_{\max }\right) / 2$. Besides that, the output of the optical channel can be written as

$h_{\text {opt }}(s)=h(s)+n_{A W G N}$,

where $n_{A W G N}$ is the additive white Gaussian noise (AWGN), and $h(\cdot)$ denotes the response of the optical channel. Finally, the inverse ellipse transform can be written as

$L(s)=\arctan \left(\frac{s-D C_{r}}{a \sqrt{1-\left(s-D C_{r}\right)^{2} / b^{2}}}\right) / \beta$,

where $D C_{r}$ denotes the DC component of the received signals.

Since E-DCO-OFDM is a nonlinear system, whereby the nonlinear transform $T(\cdot)=L\left(h_{\text {opt }}(G(E l l(\cdot)))\right)$ is performed on the realvalued sequence $\mathbf{x}$, which is Gaussian distributed with zero mean, according to Bussgang theorem and Rowe's work in [18], the output of the nonlinear transform $\tilde{x}_{D C O}[n]$ can be represented as

$$
\begin{aligned}
\tilde{x}_{D C O}[n] & =T\left(x_{D C O}[n]\right)=L\left(h_{o p t}\left(G\left(\operatorname{Ell}\left(x_{D C O}[n]\right)\right)\right)\right) \\
& =\alpha x_{D C O}[n]+n_{d}[n],
\end{aligned}
$$

where $x_{D C O}[n]$ for $n=0,1,2, \ldots, N-1$ denotes the real-valued output of IFFT, considered as a random variable following the Gaussian distribution with zero mean [10], $\alpha$ is a constant scaling factor less than 1 , and $n_{d}[n]$ is the noise and distortion component which is uncorrelated with $x_{D C O}[n]$, that is, $E\left(x_{D C O}[n] n_{d}[n]\right)=0$. The expression of $\alpha$ can be formulated as

$\alpha=\frac{E\left(T\left(x_{D C O}[n]\right) x_{D C O}[n]\right)}{\sigma_{x}^{2}}=\int T(v) v f_{x_{D C O}[n]}(v) / \sigma_{x}^{2} d v$,

where $f_{x_{D C O}[n]}(\cdot)$ is the PDF of $x_{D C O}[n]$. In addition, the variance of $n_{d}[n]$ can be derived as [18]

$$
\begin{aligned}
\sigma_{d}^{2} & =E\left(n_{d}^{2}[n]\right)-E^{2}\left(n_{d}[n]\right) \\
& =E\left(T^{2}\left(x_{D C O}[n]\right)\right)-\alpha^{2} \sigma_{x}^{2}-E^{2}\left(T\left(x_{D C O}[n]\right)\right) .
\end{aligned}
$$

At the receiver, according to the central limit theorem, the distortion component is transformed into additive Gaussian noise after a $\mathrm{N}$-point FFT operation [18]. Therefore, after the nonlinear transform $T(\cdot)$, the useful signal energy is reduced by a scaling factor of $\alpha^{2}$, and an additive Gaussian noise component with energy of $\sigma_{d}{ }^{2}$ is added. The expression of $S N R_{r}$ can be derived as

$S N R_{r}=\frac{\alpha^{2} \sigma_{x}^{2}}{\sigma_{d}^{2}}$

Since better BER performance can be achieved when $S N R_{r}$ increases according to (12), the relationship between $S N R_{r}$ and $a / b$ needs to be investigated. To optimize the performance of E-DCOOFDM, numerical approaches are employed to maximize $S N R_{r}$, whereby $b^{2}=2$, and $\gamma=2.7$. The size of IFFT $N$ is set to 512 , and the number of modulated subcarriers is 200. Besides that, the 

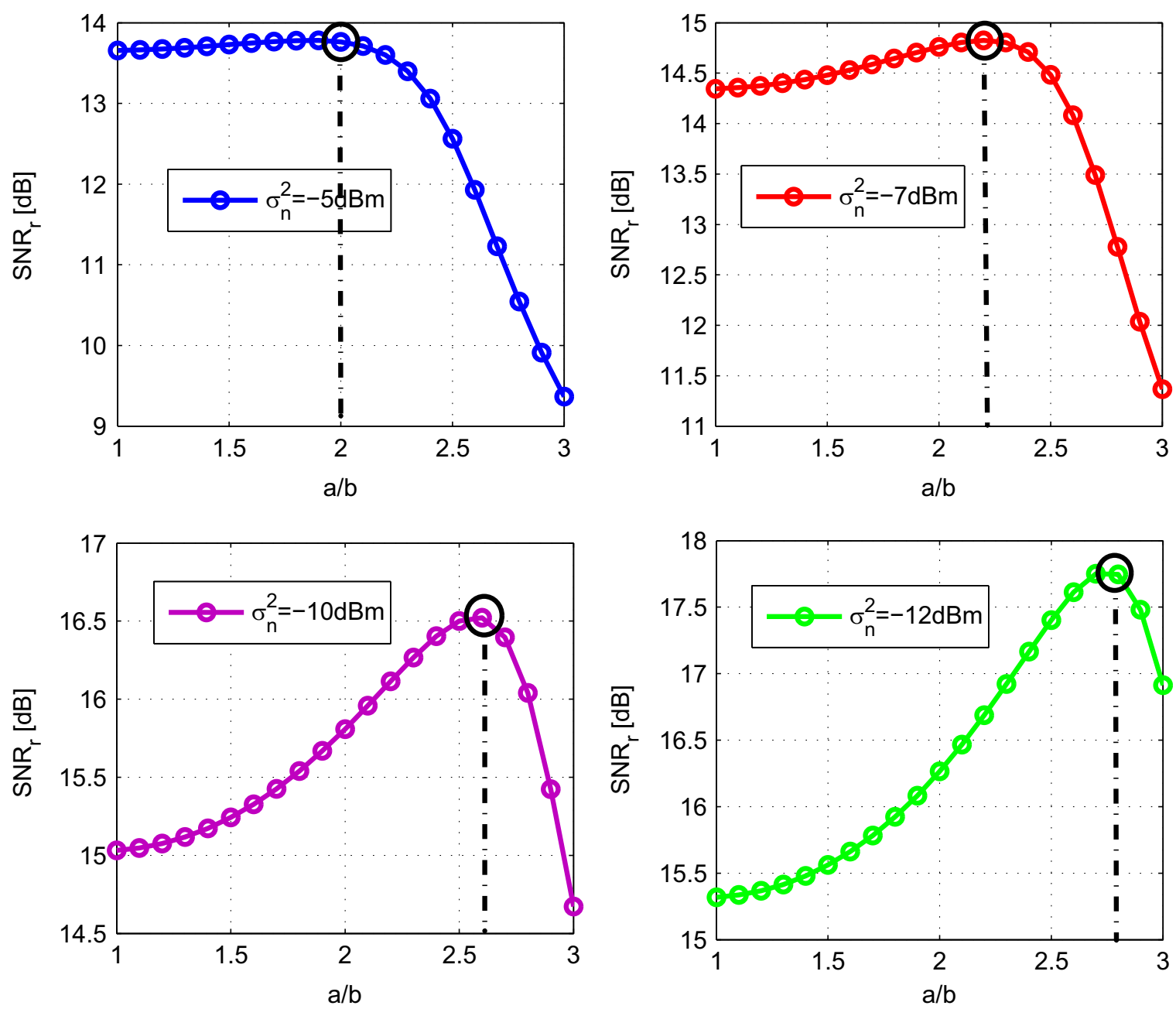

Fig. 3. $S N R_{r}$ of E-DCO-OFDM with different $a / b$ and noise energy.

simplified LED nonlinear model in [11] is applied, whereby the linear region is $[0,0.5]$, the transmitting energy of LEDs is $13.5 \mathrm{dBm}$, and AWGN channel is assumed [10].

With different values of noise energy, $S N R_{r}$ with respect to various ratios of $a / b$ are drawn according to (13)-(20) to obtain the optimal value, as shown in Fig. 3. For four different scenarios where the noise energy, denoted by ${\sigma_{n}}^{2}$, equals $-5 \mathrm{dBm},-7 \mathrm{dBm}$, $-10 \mathrm{dBm}$, and $-12 \mathrm{dBm}$, the optimal values of $a / b$ are different, which are approximately 2, 2.2, 2.6 and 2.75, respectively. Besides that, it can be observed from Fig. 3 that the optimal value of $a / b$ increases as the noise energy gets smaller, because the noise component may become less dominant than the clipping noise caused by LED nonlinear characteristics, which implies that achieving lower PAPR by enlarging $a / b$ can make the system more robust to nonlinear distortions of LEDs, therefore enhancing the system performance.

\section{Simulation results}

The complementary cumulative distribution functions (CCDFs) of PAPR in E-DCO-OFDM with different values of $a / b$ are compared with DCO-OFDM in Fig. 4, whereby 16-QAM is applied. The size of IFFT $N$ is set to 512 , and the number of modulated subcarriers is 200. For DCO-OFDM, the PAPR equals $13.5 \mathrm{~dB}$ as the CCDF approaches $10^{-3}$. While for E-DCO-OFDM with $a^{2}=10$ and $b^{2}=2$, the PAPR is about $6 \mathrm{~dB}$ less than DCO-OFDM, which makes the

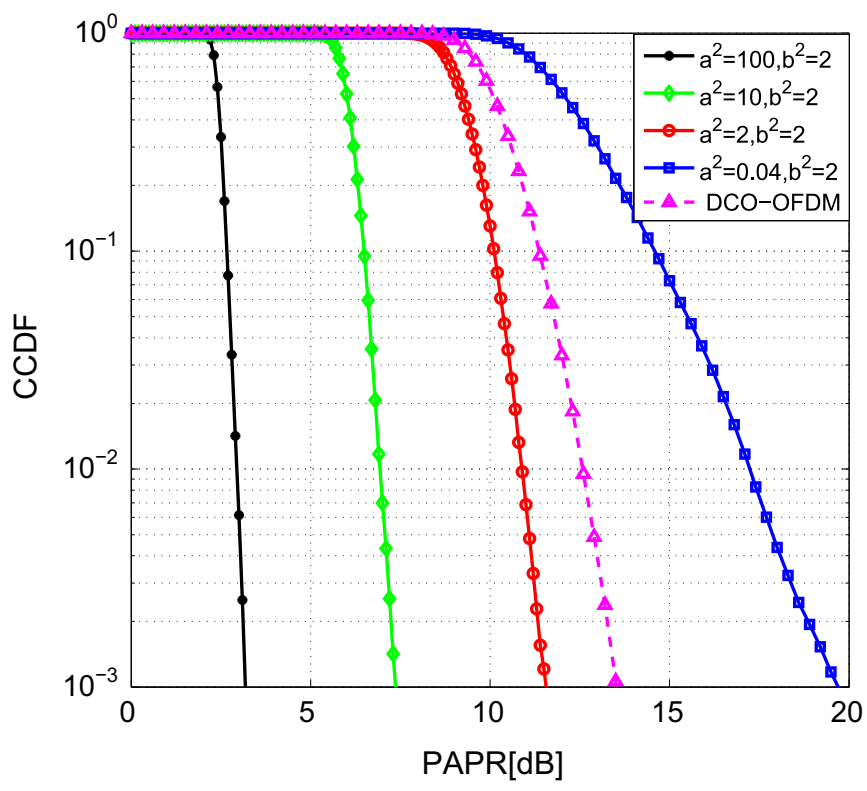

Fig. 4. CCDF of PAPR in E-DCO-OFDM with different values of $a / b$ and DCO-OFDM

system less sensitive to the nonlinear distortions of LEDs. Moreover, when comparing the CCDFs of E-DCO-OFDM with different values of $a / b$, it is indicated that the PAPR of E-DCO-OFDM gets 


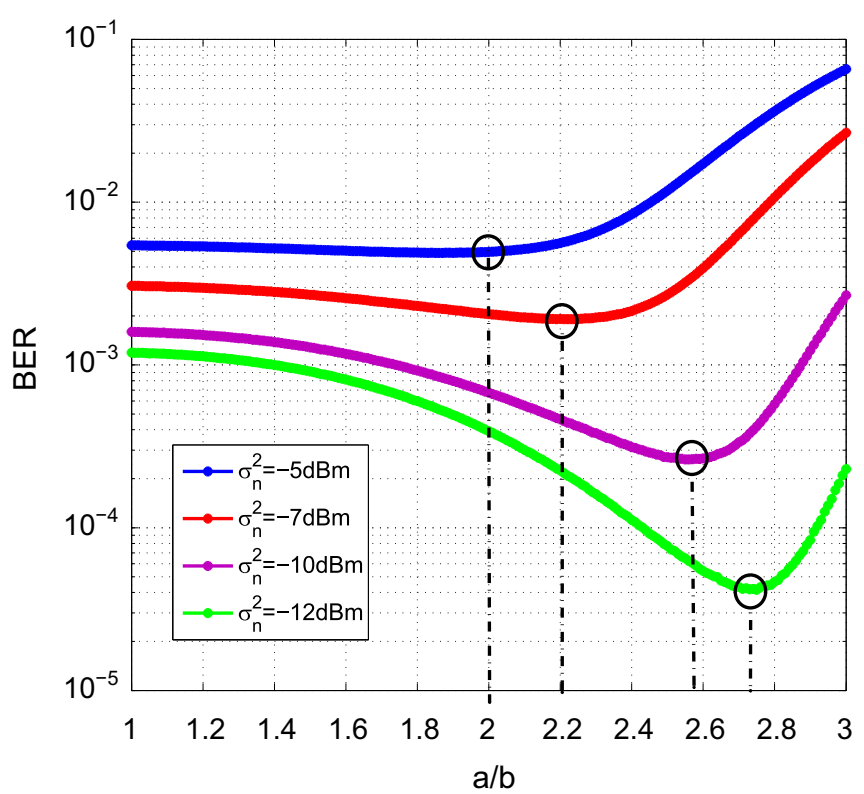

Fig. 5. BER of E-DCO-OFDM with different values of $a / b$.

smaller as the ratio of $a / b$ becomes larger, which validates our theoretical analysis in Section 3.

The BER curves of E-DCO-OFDM corresponding to different values of $a / b$ are presented under certain noise energy in Fig. 5. The simplified LED nonlinear model in [11] is employed, whereby the linear region is $[0,0.5]$, and the transmitting energy of LEDs equals $13.5 \mathrm{dBm}$. It can be implied that, for different ${\sigma_{n}}^{2}$, there is a tradeoff in performance optimization for E-DCO-OFDM. When $\sigma_{n}{ }^{2}$ equals $-5 \mathrm{dBm},-7 \mathrm{dBm},-10 \mathrm{dBm}$, and $-12 \mathrm{dBm}$, the corresponding optimal values of $a / b$ are approximately 2, 2.2, 2.6 and 2.75 , respectively. Since the optimal values of $a / b$ are in aligned with the theoretical results given in Fig. 3, our proposed optimization analysis is validated by simulations.

The BER performance of E-DCO-OFDM adopting the optimal ratios of $a / b$ in Fig. 5 is compared with DCO-OFDM and ACO-OFDM

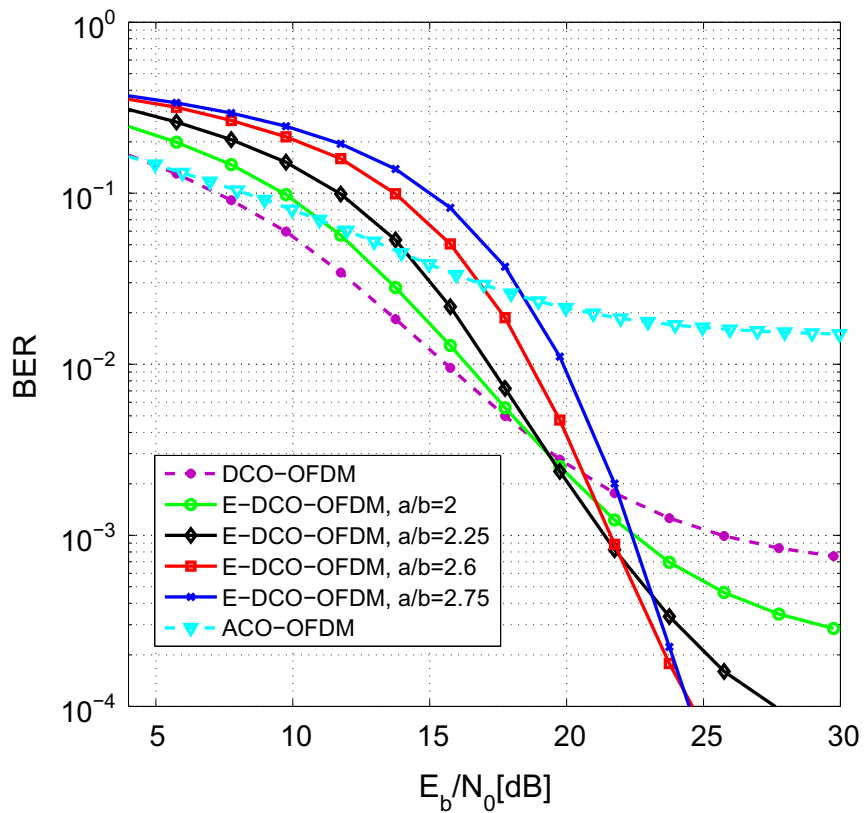

Fig. 6. BER performance comparison between the optimized E-DCO-OFDM, DCOOFDM, and ACO-OFDM. in Fig. 6, whereby the transmitting energy equals $13.5 \mathrm{dBm}$. In simulations, the same spectral efficiency is assumed, and the transmission bandwidth is set as $100 \mathrm{MHz}$. 16-QAM is used in E-DCO-OFDM and DCO-OFDM. Since the spectral efficiency of ACO-OFDM is about half of E-DCO-OFDM and DCO-OFDM when the same QAM order is employed, 256-QAM can be applied to reach the spectral efficiency approximately equal to E-DCO-OFDM and DCO-OFDM. For ACO-OFDM, there is a severe error floor when BER approaches $10^{-2}$, for the reason that the ACO-OFDM with 256-QAM is more sensitive to the clipping noise by LEDs compared with smaller QAM orders, leading to performance degradation. Besides that, at the BER of $10^{-3}$, E-DCO-OFDM with $a / b=2,2.2,2.6,2.75$ achieves significant performance gain of about $4 \mathrm{~dB}, 4.8 \mathrm{~dB}, 5 \mathrm{~dB}$, and $4 \mathrm{~dB}$ over conventional DCO-OFDM, respectively. When SNR increases, the performance gains become larger, and larger $a / b$ is needed, which is in aligned with results of Fig. 3. Besides that, when SNR is relatively low, the E-DCO-OFDM achieves no performance gain compared with DCO-OFDM, while for high SNR, E-DCO-OFDM in four different cases outperforms DCO-OFDM. The reason is that, when SNR is low, the AWGN instead of nonlinear distortion is the dominant element to effect the system performance. When SNR increases and the AWGN is low, which is also the working point for practical systems, the nonlinear distortion is dominant and the proposed scheme achieves better performance since it has lower PAPR.

Fig. 7 presents the BER performance of E-DCO-OFDM with different QAM orders, whereby the simplified LED model in [11] is used. The linear region is set to $[0,0.5]$. It is indicated that the E-DCO-OFDM with higher QAM orders is more sensitive to the clipping noise by LEDs. Therefore, in order to reach the comparable performance for E-DCO-OFDM systems with different QAM orders, the transmitting energy is $13.5 \mathrm{dBm}, 12.0 \mathrm{dBm}$, and $10.0 \mathrm{dBm}$ for 16-QAM, 64-QAM, and 256-QAM, respectively. When the BER approaches $10^{-3}$, E-DCO-OFDM with 16-QAM achieves $5.5 \mathrm{~dB}$ performance gain over 64-QAM, and $11.5 \mathrm{~dB}$ gain over 256-QAM. Thus, the E-DCO-OFDM system with larger QAM orders suffers from performance loss compared with smaller QAM orders, since it is more sensitive to noise. The performance comparison of E-DCO-OFDM with different IFFT sizes is presented in Fig. 8, whereby $16-\mathrm{QAM}$ is used. The linear region of the LED model is $[0,0.5]$, and the transmitting energy is $13.5 \mathrm{dBm}$. For different IFFT

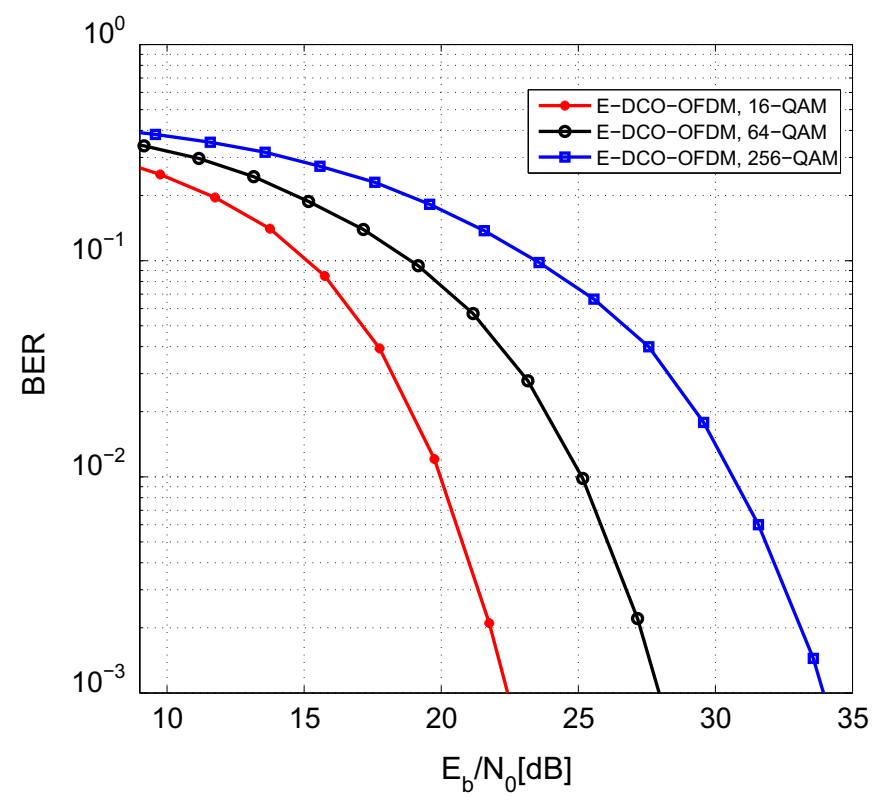

Fig. 7. BER performance comparison of E-DCO-OFDM with different QAM orders. 


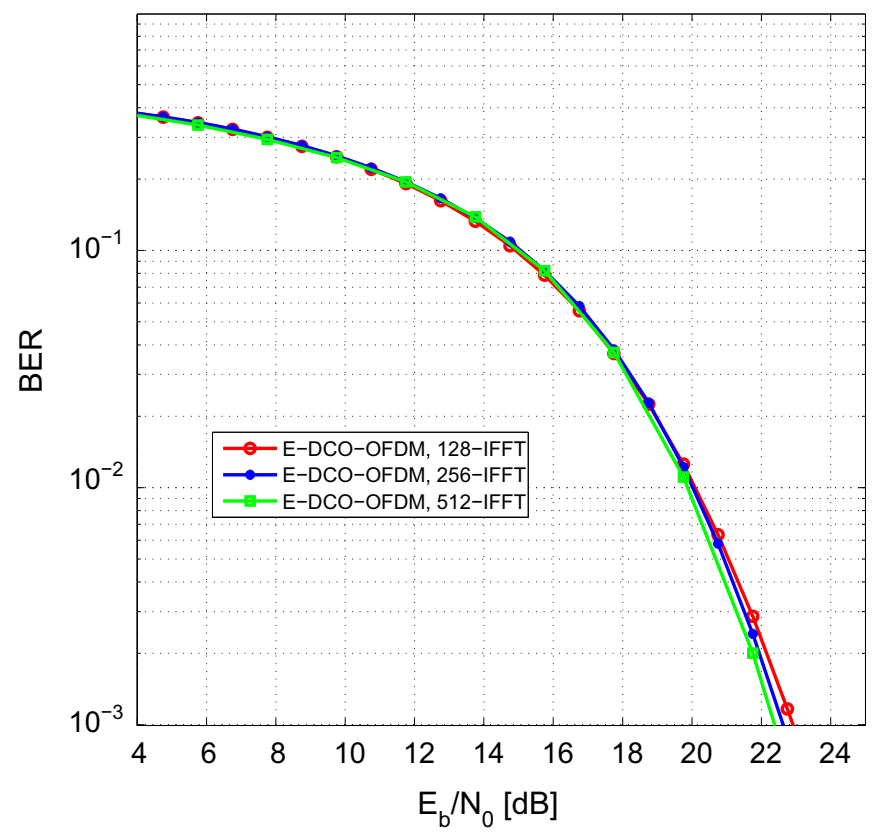

Fig. 8. BER performance comparison of E-DCO-OFDM with different subcarrier number.

sizes, the BER performance of E-DCO-OFDM is approximately the same, which indicates that the subcarrier number does not influence the BER of E-DCO-OFDM.

\section{Conclusions}

E-DCO-OFDM is proposed for visible light communications, whereby a real-valued signal is modulated onto an ellipse, and only the imaginary value from the corresponding point on the ellipse is transmitted. The PAPR of the transmitted signals reduces significantly compared with DCO-OFDM, which improves the BER performance considerably when the nonlinear transfer characteristics of LEDs is considered. Besides that, the performance optimization for E-DCO-OFDM is investigated, whereby the expressions of PAPR and BER are formulated to obtain the optimal ratio between major radius and minor radius of the ellipse. Simulations are carried out to validate the analytical results. It is indicated that E-DCO-OFDM with the optimal ratio between major radius and minor radius could achieve a considerable performance gain over DCO-OFDM.

\section{Acknowledgment}

This work was supported by National Key Basic Research Program of China (Grant no. 2013CB329203), National Nature Science Foundation of China (Grant no. 61271266), Shenzhen Visible Light Communication System Key Laboratory (Grant no. ZDSYS20140512114229398), Shenzhen Wireless over Visible Light Communication Technology Engineering Laboratory (Grant no. SDRC[2012] no. 1440) and Shenzhen Peacock Plan (Grant no. 1108170036003286).

\section{References}

[1] A. Jovicic, J. Li, T. Richardson, Visible light communication: opportunities, challenges and the path to market, IEEE Commun. Mag. 51 (12) (2013) 26-32.

[2] A.M. Khalid, G. Cossu, R. Corsini, R. Choudhury, E. Ciaramella, 1-Gb/s transmission over a phosphorescent white LED by using rate-adaptive discrete multitone modulation, IEEE Photon. J. 4 (5) (2012) 1465-1473.

[3] Q. Wang, Z. Wang, L. Dai, Iterative receiver for hybrid asymmetrically clipped optical OFDM, J. Lightwave Technol. 32 (22) (2014) 3869-3875.

[4] Q. Wang, Z. Wang, L. Dai, Asymmetrical hybrid optical OFDM for visible light communication systems with dimming control, IEEE Photon. Technol. Lett. 27 (9) (2015) 947-977.

[5] R. Jiang, Q. Wang, F. Wang, L. Dai, Z. Wang, An optimal scaling scheme for DCOOFDM based visible light communications, Opt. Commun. 356 (2015) 136-140.

[6] J.B. Carruthers, J.M. Kahn, Multiple-subcarrier modulation for nondirected wireless infrared communication, IEEE J. Sel. Areas Commun. 14 (3) (1996) $538-546$.

[7] J. Armstrong, A.J. Lowery, Power efficient optical OFDM, Electron. Lett. 42 (6) (2006) 370-372.

[8] S.C.J. Lee, S. Randel, F. Breyer, A.M.J. Koonen, PAM-DMT for intensity-modulated and direct-detection optical communications, IEEE Photon. Technol. Lett. 21 (23) (2009) 1749-1751.

[9] Q. Wang, C. Qian, X. Guo, Z. Wang, D.G. Cunningham, I.H. White, Layered ACOOFDM for intensity-modulated direct-detection optical wireless transmission, Opt. Express 23 (9) (2015) 12382-12393.

[10] S.D. Dissanayake, J. Armstrong, Comparison of ACO-OFDM, DCO-OFDM and ADO-OFDM in IM/DD systems, J. Lightwave Technol. 31 (7) (2013) 1063-1072.

[11] H. Zhang, Y. Yuan, W. Xu, PAPR reduction for DCO-OFDM visible light communications via semidefinite relaxation, IEEE Photon. Technol. Lett. 26 (17) (2014) 1718-1721.

[12] K. Bandara, P. Niroopan, Y. Chung, PAPR reduced OFDM visible light communication using exponential nonlinear companding, in: Proceedings of IEEE COMCAS, October 2013, pp. 1-5.

[13] W.O. Popoola, Z. Ghassemlooy, B.G. Stewart, Pilot-assisted PAPR reduction technique for optical OFDM communication, J. Lightwave Technol. 32 (7) (2014) 1374-1382.

[14] J.G. Doblado, A.C. Oria, V.B. Lecuyer, P. Lopez, D.P. Calderon, Cubic metric reduction for DCO-OFDM visible light communication systems, J. Lightwave Technol. 33 (10) (2015) 1971-1978.

[15] S.C. Thompson, A.U. Ahmed, J.G. Proakis, J.R. Zeidler, M.J. Geile, Constant envelope OFDM, IEEE Trans. Commun. 56 (8) (2015) 1300-1312.

[16] Z. Wang, Q. Wang, S. Chen, L. Hanzo, An adaptive scaling and biasing scheme for OFDM-based visible light communication systems, Opt. Express 22 (10) (2014) 12707-12715.

[17] S. Dimitrov, H. Haas, Information rate of OFDM-based optical wireless communication systems with nonlinear distortion, J. Lightwave Technol. 31 (6) (2013) 918-929.

[18] D. Tsonev, S. Sinanovic, H. Haas, Complete modeling of nonlinear distortion in OFDM-based optical wireless communication, J. Lightwave Technol. 31 (18) (2013) 3064-3076. 